\title{
Molecular mapping of the Pi2/9 allelic gene Pi2-2 conferring broad-spectrum resistance to Magnaporthe oryzae in the rice cultivar Jefferson
}

Nan Jiang ${ }^{1,2}$, Zhiqiang $\mathrm{Li}^{1,2}$, Jun Wu' $\mathrm{W}^{1}$ Y Yue Wang ${ }^{1}$, Liqun $\mathrm{Wu}^{1}$, Suhua Wang ${ }^{1}$, Dan Wang ${ }^{1}$, Ting Wen ${ }^{1}$, Yi Liang ${ }^{1}$, Pingyong Sun ${ }^{1}$, Jinling Liu' ${ }^{1,2}$, Liangying Dai ${ }^{1}$, Zhilong Wang ${ }^{1}$, Chao Wang ${ }^{3}$, Meizhong Luo ${ }^{3}$, Xionglun Liu ${ }^{*}$ and Guo-Liang Wang ${ }^{1,2,4^{*}}$

\begin{abstract}
Background: Utilization of broad-spectrum resistance $(R)$ genes is an effective and economical strategy to control the fungal pathogen Magnaporthe oryzae, the causal agent of the rice blast disease. Among the cloned blast resistance genes, Pi9, Pi2 and Piz-t confer broad-spectrum resistance to diverse M. oryzae isolates and were isolated from the Pi2/9 locus on chromosome 6. Identification and isolation of additional $R$ genes with different resistance spectra from this locus will provide novel genetic resources for better control of this important rice disease.

Results: In this study, we identified a dominant $R$ gene, Pi2-2, at the Pi2/9 locus from Jefferson, an elite U.S. rice cultivar, through genetic and physical mapping. Inoculation tests showed that Jefferson has different resistant specificities to M. oryzae isolates compared rice lines with the Pi9, Pi2 and Piz-t genes. Fine mapping delimited Piz-2 to a 270-kb interval between the markers AP5659-3 and RM19817, and this interval contains three nucleotide-binding site-leucine-rich repeat (NBS-LRR) genes in the Nipponbare genome. Five bacterial artificial chromosome (BAC) clones spanning the region were identified, and a BAC contig covering the Pi2-2 locus was constructed.
\end{abstract}

Conclusions: We identified a new allelic gene at the Pi2/9 locus and fine-mapped the gene within a 270-kb region. Our results provide essential information for the isolation of the Pi2-2 gene and tightly linked DNA markers for rice blast resistance breeding.

Keywords: Rice blast, Resistance gene, Mapping, BAC clones, Pi2/9 locus

\section{Background}

Rice is the staple food for more than half people of the world, and the demand is increasing because of the expanding rice-eating population, particularly in many developing countries in Africa and Asia. However, rice production is severely affected by various biotic and abiotic stresses (Khush and Jena 2009). Rice blast, caused by the fungal pathogen Magnaporthe oryzae, is one of

\footnotetext{
*Correspondence: xionglun@yahoo.com; wang.620@osu.edu

${ }^{1}$ Hunan Provincial Key Laboratory of Crop Germplasm Innovation and Utilization, College of Agronomy, College of Bio-Safety Science and Technology, Hunan Agricultural University, Changsha 410128, China ${ }^{2}$ State Key Laboratory for Biology of Plant Diseases and Insect Pests, Institute of Plant Protection, Chinese Academy of Agricultural Sciences, Beijing 100193, China

Full list of author information is available at the end of the article
}

the major limitations, and usually causes $10-30 \%$ yield loss in rice production when a rice blast epidemic occurs (Talbot 2003; Skamnioti and Gurr 2009). Use of host resistance is an effective and economical way to control the blast disease (Khush and Jena 2009). To date, over 80 blast resistance genes have been identified, and are distributed on 11 rice chromosomes except chromosome 3 (Liu et al. 2010; Yang et al. 2009). So far, 21 have been cloned (Pib, Pita, Pi9, Pi2, Piz-t, Pid2, Pi36, Pi37, Pik-m, Pit, Pi5, Pid3, pi21, Pb1, Pish, Pik, Pik-p, Pi54, Pia, NLS1 and Pi25). Interestingly, most of them are NBSLRR genes except $P i-d 2$ and pi21 (Wang et al. 1999; Bryan et al. 2000; Qu et al. 2006; Zhou et al. 2006; Chen et al. 2006; Liu et al. 2007; Lin et al. 2007; Ashikawa et al. 2008; Hayashi and Yoshida. 2009; Lee et al. 2009; 
Shang et al. 2009; Fukuoka et al. 2009; Hayashi et al. 2010; Takahashi et al. 2010; Zhai et al. 2011; Yuan et al. 2011; Sharma et al. 2005; Okuyama et al. 2011; Tang et al. 2011; Chen et al. 2011). Pi-d2 encodes a receptorlike kinase protein with a predicted extracellular domain of a bulb-type mannose-specific binding lectin (B-lectin) and an intracellular serine-threonine kinase domain (Chen et al. 2006). Pi21 encodes a proline-rich protein that includes a putative heavy metal-binding domain and protein-protein interaction motifs. The resistant allele pi21 carrying deletions in the proline-rich motif can reduce blast infection rate (Fukuoka et al. 2009). Pik, $P i k-m$ and $P i k-p$ are located at the locus of Pik on chromosome 11, and interestingly, each of them requires two independent NBS-LRR genes for the blast resistance (Zhai et al. 2011; Ashikawa et al. 2008; Yuan et al. 2011). Similarly, both Pi5 and Pia also require two NBS-LRR members for their resistance function (Lee et al. 2009; Okuyama et al. 2011).

At least eight blast resistance genes were identified from the Pi2/9 locus, which is located on the short arm and near the centromere of chromosome 6. Among them, Pi9, Pi2 and Piz-t were successfully cloned (Qu et al. 2006; Zhou et al. 2006). Pi26(t) (Wu et al. 2005), Pigm( $t$ ) (Deng et al. 2006), Piz(t) (Fjellstrom et al. 2006), Pi4O( $t$ ) (Jeung et al. 2007) and Pi50(t) (Zhu et al. 2012) are in the process of being cloned by different laboratories. Interestingly, most of them confer broadspectrum resistance to diverse $M$. oryzae races or isolates. The near isogenic line C101A51 carrying Pi2 is resistant to 455 isolates collected from Philippines and most of the 792 isolates from China (Chen et al. 1996, 1999). The Pi9-bearing line, 75-1-127, is resistant to 43 isolates collected from 13 different countries (Liu et al. 2002). Piz- $t$ and Pigm from Toride and Gumei4, respectively, are resistant to more than $90 \%$ of tested isolates from China and Thailand (Shen et al. 2003). The nearisogenic line containing Pi5O(t) is incompatible to $97.7 \%$ of the 523 isolates from different regions of China (Zhu et al. 2012). However, the underlying mechanism of broad-spectrum resistance of these genes is still not well understood.

Jefferson, a long-grain tropical japonica cultivar grown in the southern U.S., has retained its resistance to blast since its first use in 1997 (McClung et al. 1997; Skamnioti and Gurr 2009). It was reported that Jefferson possesses three blast resistance genes, $P i z(t), P i-d(t)$ and $P i-k^{h}(t)$, based on its disease reactions (McClung et al. 1997). Our preliminary observation showed that Jefferson was immune in the blast nursery of Taojiang County, Hunan Province, China, which contained 11 major $M$. oryzae races including ZC9, ZC11, ZE3, ZB29, ZG1, ZB25, ZB31, ZB13, ZC7, ZA9, and ZF1 (unpublished). To determine the genetic basis of broad-spectrum resistance in Jefferson, we performed greenhouse inoculations with individual isolates and genetic analysis using an $F_{2}$ population derived from a cross between Jefferson and the susceptible cultivar CO39. We identified a dominant $R$ gene in Jefferson on chromosome 6 at the Pi2/9 locus, named Pi2-2. Allelism analysis indicated that Pi2-2 is tightly linked or allelic to Pi9. We constructed a BAC contig in the genomic region and fine-mapped the gene within a region approximately $270 \mathrm{~kb}$. These data will facilitate both the positional cloning of the $R$ gene and molecular breeding programs of rice blast resistance.

\section{Results}

Resistance spectrum of Jefferson to $28 M$. Oryzae isolates

To test the resistance spectrum of Jefferson, we inoculated the cultivar with $28 \mathrm{M}$. oryzae isolates collected from six countries, and the inoculation results are summarized in Additional file 1: Table S1. Three known broad-spectrum resistant cultivars, Tianye carrying Pi2-1 and Pi51 (Wang et al. 2012), XZ3150 carrying Pi47 and Pi48 (Huang et al. 2011), and 75-1-127 carrying Pi9 (Qu et al. 2006) were used as resistance controls and the highly susceptible cultivar CO39 was used as a susceptible control. Interestingly, Tianye was resistant to all the isolates and Jefferson was only susceptible to the blast isolate RB11 from Japan. XZ3150 was susceptible to three isolates (236-1, RB6 and ROR1) and 75-1-127 was susceptible to two isolates (ROR1 and X2007A-7). By contrast, the susceptible control cultivar $\mathrm{CO} 39$ was susceptible to 27 of all 28 tested isolates. These results indicate that Jefferson confers broad-spectrum resistance to M. oryzae.

\section{Resistance to $M$. oryzae isolate $\mathbf{3 1 8 - 2}$ is controlled by a single dominant locus in Jefferson}

The M. oryzae isolate 318-2 from Hunan Province of China was used for genetic analysis of the blast resistance in Jefferson. We developed the $F_{2}$ population derived from a cross between Jefferson and CO39. All the $\mathrm{F}_{1}$ plants were resistant to 318-2 (32R:0S), indicating that the dominant inheritance of the $R$ gene in Jefferson. The segregation of resistant and susceptible individuals in the $F_{2}$ population fitted a ratio of 3:1 (194R:60S, $\mathrm{X} 2=0.257,0.5<\mathrm{P}<0.9$ ), suggesting that the resistance to $318-2$ is controlled by a single dominant $R$ gene in Jefferson. We designated this $R$ gene in Jefferson as Pi2-2.

\section{Pi2-2 is tightly linked or allelic to Pi9 on chromosome 6}

Previous research reported that there are three blast resistance genes, $P i z(t), P i-d(t)$ and $P i-K^{h}(t)$, in Jefferson (McClung et al. 1997). Piz(t) is located on chromosome 6 near the Pi2/9 locus (Fjellstrom et al. 2006). Pi-d(t) and $P i-k^{h}(t)$ are located on chromosome 11. Therefore, we selected 25 SSR markers around the Pi2/9 and Pi-k loci 
for linkage analysis. Twenty highly resistant and twenty highly susceptible individuals from the $F_{2}$ population of the Jefferson $\times \mathrm{CO} 39$ cross were genotyped with the polymorphic markers. No marker around the $P i-k^{h}$ locus cosegregated with the resistance to $318-2$. But two polymorphic SSR markers around Pi2/9, RM7178 and RM7311 (Table 1), were associated with the resistance, indicating that Pi2-2 is located on chromosome 6.

Previous studies showed that Pi2 and Piz- $t$ are tightly linked to Pi9 (Zhou et al. 2006, 2007) and Piz(t) is allelic or tightly linked to Piz-t (Hayashi et al. 2004). However, the exact location of $\mathrm{Piz}(t)$ has not been determined yet. To understand the linkage relationship between Pi2-2 and the $R$ genes in the same region, we developed an $\mathrm{F}_{2}$ population from a cross between Jefferson and Pi9carrying line 75-1-127 for allelism test. A total of $637 \mathrm{~F}_{2}$ individuals were inoculated with $M$. oryzae isolate $318-2$, which was incompatible to both Jefferson and 75-1-127, to observe the phenotype segregation. No susceptible plant was found in $637 \mathrm{~F}_{2}$ individuals, suggesting that Pi2-2 is tightly linked or allelic to the Pi9 gene.

\section{Jefferson shows different resistance spectrum with the cultivars carrying other $R$ genes at the Pi2/9 locus}

Previous research showed that the three cloned $R$ genes at $\mathrm{Pi} 2 / 9$ locus have different resistance spectra. $75-1-127$ (Pi9) was susceptible to ROR1, a M. oryzae strain from Korea. The isolate CHNOS60-2-3 from China could distinguish C101A51 (Pi2) and Toride (Piz-t) resistance specificities (Zhou et al. 2006). However, Jefferson was immune to both of them (Table 2). In the inoculations with 28 blast isolates (Additional file 1: Table S1), Jefferson and 75-1-127 also have different resistance spectra. In addition, another two isolates from Hunan Province, China, showed different reactions to Jefferson and Toride (Piz-t) or 5173 (Pi2). These results suggest that $P i 2-2$ is a different $R$ gene at the Pi2/9 locus. However, isogenic lines with all the $R$ genes at the Pi2/9 locus should be used in inoculations with different isolates to confirm the conclusion.

\section{Fine mapping and in silico mapping of the Pi2-2 gene}

To finely map the Pi2-2 gene, another 14 SSR markers were used, and four of them exhibited polymorphism between the two parental lines (Table 1). A total of 583 susceptible individuals from the Jefferson $\times \mathrm{CO}_{3} \mathrm{~F}_{2}$ population were genotyped with these polymorphic markers. Finally, the Pi2-2 gene was delimited by the closest flanking markers RM19817 and AP5659-3, with one and three recombinant events detected, respectively (Figure 1A). The markers RM7178 and AP5659-5 co-segregated with Pi2-2 in all 583 susceptible plants. The physical distance between the closest flanking markers, RM19817 and AP5659-3, was estimated to be about $270 \mathrm{~kb}$ according to the Nipponbare genome information in this region. A virtual contig map consisting of three overlapping Nipponbare BAC clones (P0491D10, P0502B12 and P0649C11) was constructed (Figure 1B). Annotation of the corresponding genomic sequence indicates that there are three NBS-LRR genes in this region, which are paralogs of the Pi9 gene (Figure 1C).

\section{Construction of a BAC contig covering the Pi2-2 locus}

For the cloning of the Pi2-2 gene, we constructed a genomic BAC library of Jefferson with an average insert size of $140 \mathrm{~kb}$. The tightly linked SSR markers spanning Pi2-2 were used for PCR screening of the BAC library pools. Six positive clones were identified by four SSR markers and were end-sequenced (Table 3). To confirm whether these BAC clones overlapped, the end sequences were compared with the corresponding sequences on chromosome 6 in the Nipponbare genome. The results showed that BAC clones BJ21-2-4-43 and BJ21-5-4-41 were the same. For clone BJ24-1-13, only one end was anchored at Pi2/9 locus and no homologous sequence was identified on this chromosome compared with the Nipponbare genomic sequence for the other end. The NIP (nitrite-induced protein) and PK (protein kinase) genes are the $5 /$ and $3 /$ boundaries of the Pi2/9 locus, respectively, these are highly conserved in different haplotypes (Zhou et al. 2007). Thus, the specific primer pairs NIP-2F/R (NIP-2F, 5/- TTTGGCGTGTCACATCGG-3/;

Table 1 Polymorphic SSR markers around the Pi2/9 locus used for linkage analysis

\begin{tabular}{lcccc}
\hline Markers & Forward primer (5/-3/) & Reverse primer (5/-3/) & Genomic position (bp) & Expected size (bp) \\
\hline MRG5836 & AAAAACCTAGAAAATGGGAAATG & TATAAGCCGCAGCCAAATC & $9308979-9309076$ & 98 \\
RM19817 & CCAAGGAGGTGATCCAGGAGTGC & CGGCAGAGCAGACGACATGG & $10137012-10137394$ & 383 \\
RM7178 & CCGTGAGATGGGCTACCTAC & TAACCTTCACAGCGAACGTG & $10198893-10199043$ & 151 \\
AP5659-5 & CTCCTTCAGCTGCTCCTC & TGATGACTTCCAAACGGTAG & $10357166-10357453$ & 288 \\
AP5659-3 $^{\text {a }}$ & TCTTCCTAGGGAACCAAAG & AAGTAGTTGCTGAGCCATTG & $10406597-10406825$ & 229 \\
RM7311 $^{\text {b }}$ & CGTGGCGCCTTAATCTC & AGTGGTCGTGAACTCGGAG & $11045702-11045848$ & 147 \\
\hline
\end{tabular}

${ }^{a}$ Previously reported markers in this region

b SSR markers released by Gramene database (http://www.gramene.org/db/markers/).

Genomic position and expected PCR product size for each marker were determined based on the reference sequence of rice cultivar Nipponbare released by International Rice Genome Sequencing Program (IRGSP). 
Table 2 The disease reactions of Jefferson and donors of Pi2, Piz-t and Pi9

\begin{tabular}{|c|c|c|c|c|c|c|}
\hline \multirow[t]{2}{*}{ Isolates } & \multirow[t]{2}{*}{ Origin } & \multicolumn{5}{|c|}{ Cultivars } \\
\hline & & Jefferson & 5173 (Pi2) & Toride $($ Piz- $t)$ & 75-1-127 (Pi9) & CO39 \\
\hline ROR1 & Korea & $R$ & $\mathrm{R}$ & $R$ & $\mathrm{~S}$ & $\mathrm{~s}$ \\
\hline CHNOS60-2-3 & China & $\mathrm{R}$ & S & $\mathrm{R}$ & $\mathrm{R}$ & $S$ \\
\hline $236-1$ & China & $\mathrm{R}$ & $\mathrm{R}$ & $\mathrm{S}$ & $\mathrm{R}$ & $\mathrm{s}$ \\
\hline X2007A-7 & China & $\mathrm{R}$ & $\mathrm{R}$ & $\mathrm{s}$ & S & $S$ \\
\hline
\end{tabular}

$\mathrm{R}$ and $\mathrm{S}$ denote resistant and susceptible reaction, respectively.

NIP-2R, 5/-TGGAGCGGAGACAGAGTGG-3/) and PK1F/R (PK-1F, 5/-CGTTCACTGACTTCCCTTTCCC3/; PK-1R, 5/-TCCGCATCGCCGTCTTCTG-3/), designed based on the NIP and $P K$ sequences, were employed for detecting the relative location of the five $\mathrm{BAC}$ clones at the Pi2/9 locus. The PCR results showed BJ2-4-1-13 contained the $P K$ gene. A contig map consisting of $5 \mathrm{BAC}$ clones (B)2-7-10-8, BJ21-2-3-10, BJ21-7-3-51, BJ21-2-4-43 and BJ2-4-1-13) was constructed that covered both Pi2-2 and the whole Pi2/9 locus in Jefferson (Figure 2).

\section{Discussion}

Many plant disease resistance genes are located in complex clusters in which multiple copies of closely related sequences are formed through gene duplication and uneven crossing over. Allelic genes in different genetic backgrounds have evolved to carry diverse resistance specificities due to exposure of these loci to different pathogen populations. In rice, over half of the identified blast resistance genes are clustered at different loci, especially on chromosomes 6,11 and 12 . The Pi2/9 locus is a region with at least eight $R$ genes (Yang et al. 2009;
Zhu et al. 2012), and contains several NBS-LRR type genes in both cultivated and wild rice lines (Zhou et al. 2007; Dai et al. 2010). Three $R$ genes at this locus have been successfully isolated. The paralog NBS2Pi9 is the Pi9 gene, and the paralogs NBS4-Pi2 and NBS4-Piz- $t$ are the Pi2 and Piz- $t$ genes, respectively (Zhou et al. 2006). In our study, three candidate NBS-LRR genes (NBS-LRR1, NBS-LRR2 and NBS-LRR3) at the Pi2/9 locus were identified for Pi2-2 according to the sequence of Nipponbare genome. However, the Nipponbare genome did not fully reflect the structure of the Pi2-2 locus in Jefferson. Thus, sequence analysis of the BAC clones of Jefferson covering Pi2-2 and complementation test of candidate genes are necessary for determining which NBS-LRR gene is Pi2-2.

Three blast resistance genes, $P i z(t), P i-d(t)$ and $P i k_{-}{ }^{h}(t)$, were reported in Jefferson (McClung et al. 1997). Pi-d(t) and Pik $^{-}{ }^{h}(t)$ are tightly linked on chromosome 11. Piz(t) was originally reported in the U.S. rice cultivar Zenith (Kiyosawa 1967), and has been widely introduced into different cultivars by rice breeders (Conaway-Bormans et al. 2003). Piz(t) was mapped on the short arm of

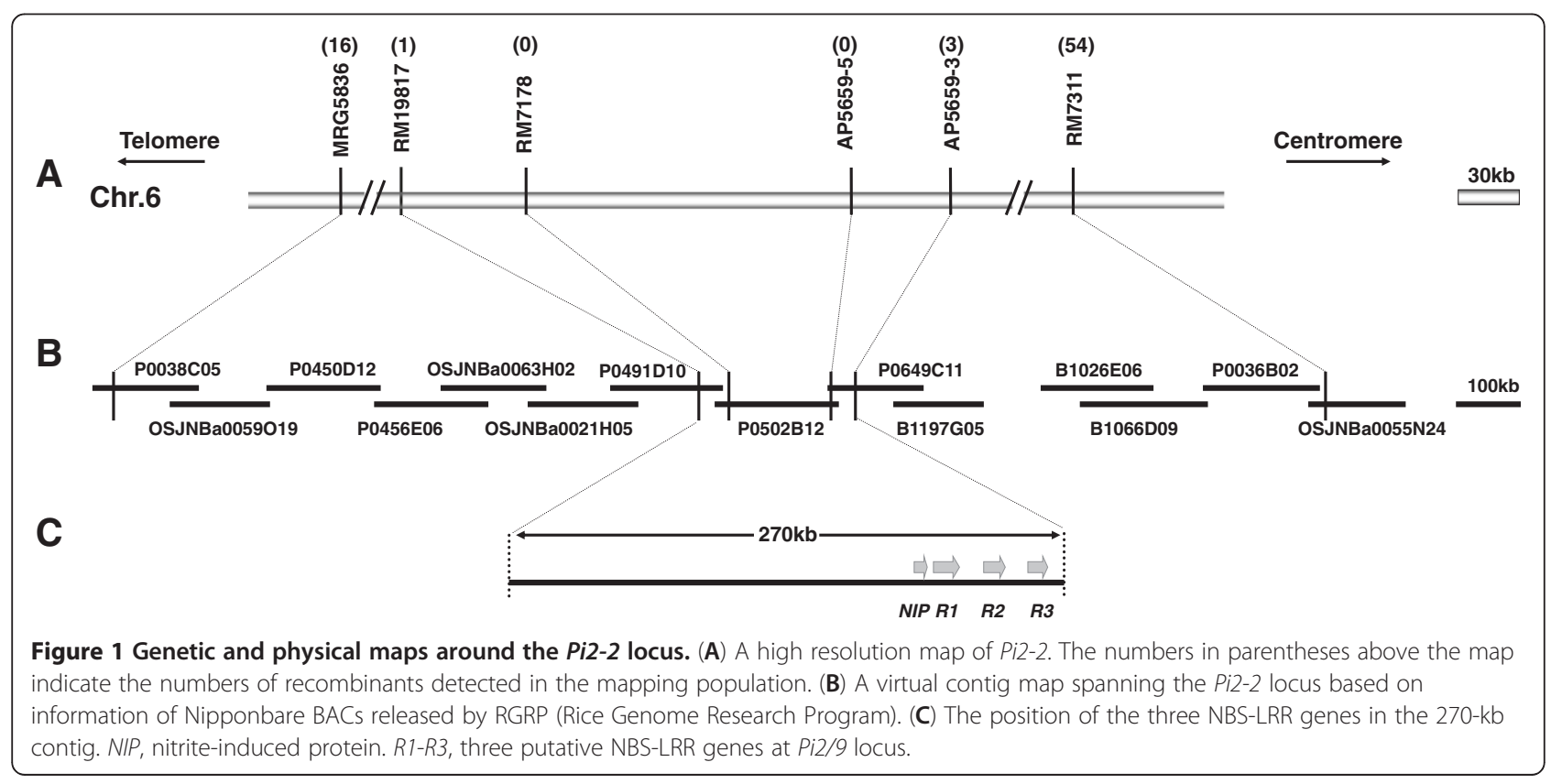


Table 3 PCR screening of positive BAC clones from the Jefferson BAC library

\begin{tabular}{|c|c|c|c|c|c|c|c|c|}
\hline \multirow[t]{2}{*}{ BAC clones } & \multicolumn{5}{|c|}{ Markers } & \multirow[b]{2}{*}{ PK } & \multicolumn{2}{|c|}{ Physical locations } \\
\hline & RM19817 & RM7178 & AP5659-5 & NIP & AP5659-3 & & Start & Stop \\
\hline BJ2-7-10-8 & + & + & - & - & - & - & 10076481 & 10204423 \\
\hline BJ21-2-3-10 & - & + & + & + & - & - & 10148738 & 10392837 \\
\hline BJ21-7-3-51 & - & + & + & + & - & - & 10170474 & 10393164 \\
\hline BJ21-2-4-43 & - & - & + & + & + & - & 10212052 & 10417645 \\
\hline BJ21-5-4-41 & - & - & + & + & + & - & 10212052 & 10417645 \\
\hline BJ2-4-1-13 & - & - & - & - & + & + & 10380807 & N/A \\
\hline
\end{tabular}

+ , positive; -, negative.

The physical locations were determined by comparing the end sequences of the BAC clones with the Nipponbare genomic sequence.

chromosome 6, close to the centromere, by several groups using different cultivars (Hayashi et al. 2006; Fjellstrom et al. 2006; Conaway-Bormans et al. 2003), but the exact location has not been determined yet. Based on the fine mapping results in this study, we speculate that Pi2-2 is likely $\mathrm{Piz}(\mathrm{t})$. Our on-going cloning effort of the Pi2-2 gene will provide us the answer in the near future.

\section{Conclusions}

This study demonstrated that the rice cultivar Jefferson harbors the blast resistance gene Pi2-2 at the Pi2/9 locus on chromosome 6 . The gene was finely mapped to a 270 kb interval. A BAC contig covering Pi2-2 was constructed, which provides essential foundation for the isolation of the $R$ gene.

\section{Methods}

\section{Plant materials}

Seven rice cultivars, Jefferson, Tianye, XZ3150, 5173 (Pi2), Toride (Piz-t), 75-1-127 (Pi9) and CO39, were used in this study. $F_{1}$ and $F_{2}$ populations from a cross between Jefferson and highly susceptible cultivar CO39 were constructed for genetic analysis. The $\mathrm{F}_{2}$ population derived from a cross between Jefferson and 75-1-127 was constructed for allelism tests.

\section{Blast inoculation and disease evaluation}

The $28 \mathrm{M}$. oryzae isolates used in the study are listed in Additional file 1: Table S1. The collection sites and providers are included in the table. Rice seedlings at 3-4

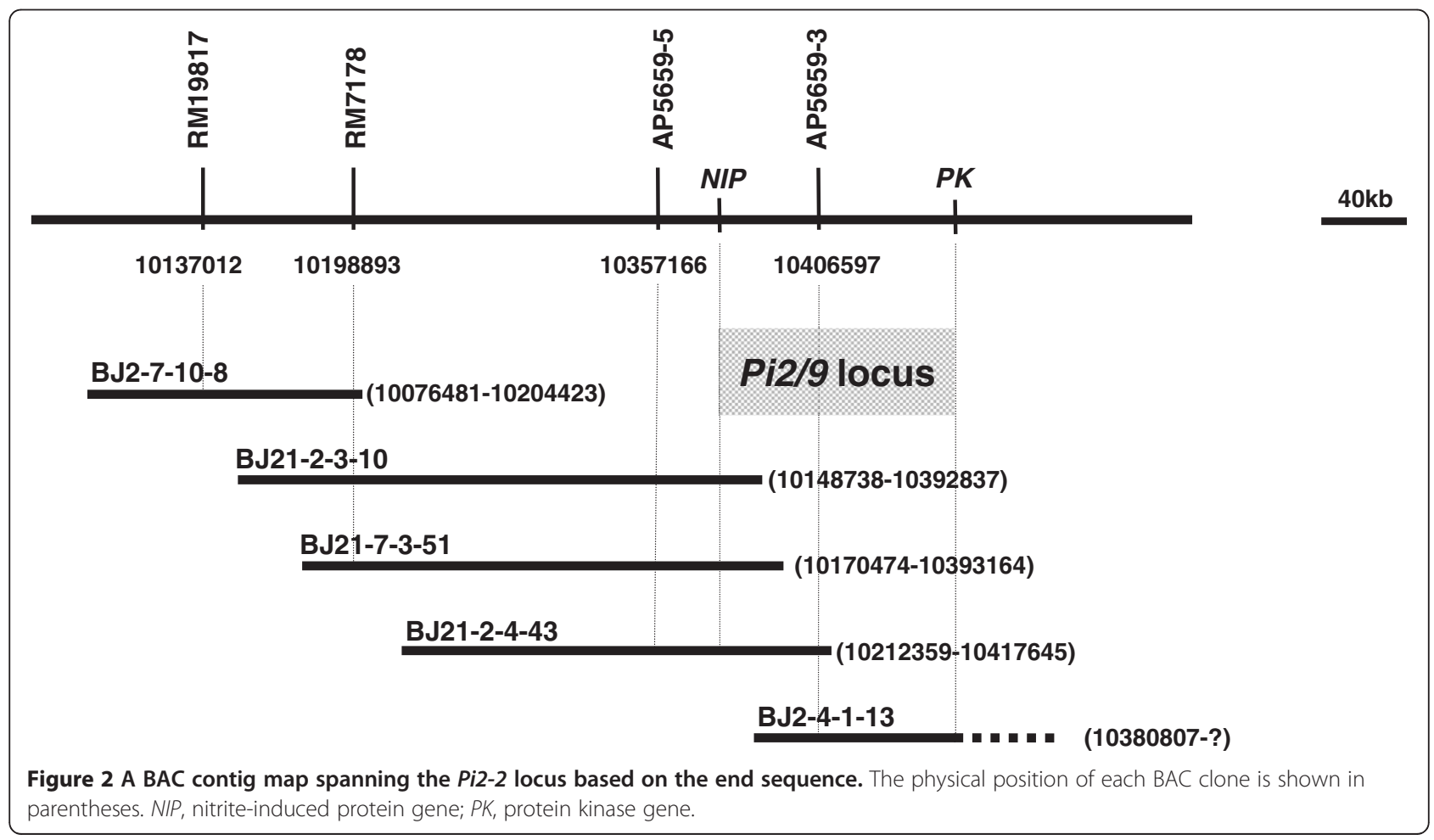


leaf-stage were spray-inoculated with $M$. oryzae spore suspensions $\left(1.5 \times 10^{5}\right.$ spores $\left./ \mathrm{ml}\right)$ and then kept in darkness at $25^{\circ} \mathrm{C}-27^{\circ} \mathrm{C}$ and over $90 \%$ relative humidity for $24 \mathrm{~h}$. The inoculated plants were subsequently kept under a 12/12 (day/night) photoperiod at the same temperature and relative humidity. Disease reaction evaluation was carried out 7 days after inoculation according to the 0-5 scoring system described by (Bonman et al. 1986).

\section{Genetic and allelism analysis}

The Jefferson $\times \mathrm{CO} 39 \quad \mathrm{~F}_{2}$ population was inoculated with the $M$. oryzae isolate $318-2$, which is avirulent to Jefferson and virulent to CO39. 318-2, which is also avirulent to 75-1-127, was employed to inoculate the Jefferson $\times 75-1-127 F_{2}$ population for allelism analysis.

\section{Genotyping and genetic mapping}

A total of 39 SSR markers spanning the Pi2/9 and Pik loci were used for the polymorphism survey between Jefferson and CO39. Six polymorphic SSR markers spanning the Pi2/9 locus were used for preliminary and fine mapping of the $R$ gene in Jefferson (Table 1). The genomic DNA of 20 highly resistant and 20 susceptible $F_{2}$ individuals, which were phenotypically confirmed in the $\mathrm{F}_{3}$ generation, were extracted from leaves for segregation analysis (Saghai-Maroof et al. 1984). All PCRs began with a denaturation step of $94^{\circ} \mathrm{C} / 4 \mathrm{~min}$, followed by 35 cycles of (A) $94^{\circ} \mathrm{C} / 30 \mathrm{sec}, 55^{\circ} \mathrm{C} / 30 \mathrm{sec}, 72^{\circ} \mathrm{C} / 30 \mathrm{sec}$, with a final extension step of $72^{\circ} \mathrm{C} / 7 \mathrm{~min}$. Linkage analysis was performed using the MAPMAKER/V3.0 using all highly susceptible individuals.

\section{Physical mapping of the Pi2-2 locus}

The physical positions of the markers tightly linked to Pi2-2 locus were determined based on the genome of Nipponbare using the BLAST program on Gramene (http://www.gramene.org/Multi/blastview) (Jaiswal et al. 2006). The genomic sequences flanked by the markers RM19817 and AP5659-3 were annotated using the Rice Genome Annotation Project (http://rice.plantbiology. msu.edu/) (Ouyang et al. 2007) and Rice Genome Automated Annotation System (http://ricegaas.dna.affrc.go.jp/) (Sakata et al. 2002).

\section{Construction of the BAC library of Jefferson}

The genomic BAC library of Jefferson was constructed using the method described by (Luo and Wing 2003). The markers tightly linked to Pi2-2 were used for screening of positive clones from the BAC pools. The contig map spanning the Pi2-2 locus was constructed based on the end sequencing results of the positive BAC clones.

\section{Additional file}

Additional file 1: Table S1. Disease reaction of Jefferson and other 4 cultivars to $28 \mathrm{M}$. oryzaeisolates collected from different regions.

\section{Competing interests}

The authors declare that they have no competing interests.

\section{Authors' contributions}

$\mathrm{NJ}$ and ZL contributed equally to this work. NJ carried out resistance spectrum analysis, allelism analysis, genetic analysis, molecular mapping, construction of the BAC contig map and wrote the manuscript; ZL carried out molecular mapping, construction of the BAC contig map; JW carried out spectrum analysis and allelism analysis; YW participated in molecular mapping. LW carried out resistance spectrum analysis; SW carried out resistance spectrum analysis; DW carried out resistance spectrum analysis; TW carried out resistance spectrum analysis; YL participated in molecular mapping; PS carried out carried out genetic analysis; JL participated in the design of the study; LD participated in the design of the study; ZW participated in experimental designing; CW constructed the BAC library of Jefferson; ML participated in the design of the study and constructed the BAC library of Jefferson; $\mathrm{XL}$ designed the research and wrote the manuscript; GW designed the research and wrote the manuscript. All authors read and approved the final manuscript.

\section{Acknowledgements}

This work was financially supported by the National Natural Science Foundation of China (31171526 and 30571063), Hunan Provincial Natural Science Foundation (06JJ10006) and the US National Science Foundation to GLW (IOS \#1120949).

\section{Author details}

${ }^{1}$ Hunan Provincial Key Laboratory of Crop Germplasm Innovation and Utilization, College of Agronomy, College of Bio-Safety Science and Technology, Hunan Agricultural University, Changsha 410128, China. ${ }^{2}$ State Key Laboratory for Biology of Plant Diseases and Insect Pests, Institute of Plant Protection, Chinese Academy of Agricultural Sciences, Beijing 100193, China. ${ }^{3}$ State Key Laboratory of Crop Genetic Improvement, Huazhong Agricultural University, Wuhan 430070, China. ${ }^{4}$ Department of Plant Pathology, Ohio State University, Columbus, Ohio 43210, USA.

Received: 27 April 2012 Accepted: 27 September 2012 Published: 3 October 2012

\section{References}

Ashikawa I, Hayashi N, Yamane H, Kanamori H, Wu J, Matsumoto T, Ono K, Yano M (2008) Two adjacent nucleotide-binding site-leucine-rich repeat class genes are required to confer Pikm-specific rice blast resistance. Genetics 180:2267-2276

Bonman JM, Vergel De Dios TI, Khin MM (1986) Physiologic specialization of pyricularia oryzae in the Philippines. Plant Dis 70:767-769

Bryan GT, Wu KS, Farrall L, Jia Y, Hershey HP, McAdams SA, Donaldson GK, Tarchini R, Valent B (2000) A single amino acid difference distinguishes resistant and susceptible alleles of the rice blast resistance gene Pi-ta. Plant Cell 12:2033-2046

Chen D, Zeigler RS, Ahn SW, Nelson RJ (1996) Phenotypic characterization of the rice blast resistance gene Pi2(t). Plant Dis 80:52-56

Chen DH, dela Vina M, Inukai T, Mackill DJ, Ronald PC, Nelson RJ (1999) Molecular mapping of the blast resistance gene, Pi44(t), in a line derived from a durably resistant rice cultivar. Theor Appl Genet 98:1046-1053

Chen J, Shi Y, Liu W, Chai R, Fu Y, Zhuang J, Wu J (2011) A Pid3 allele from rice cultivar Gumei2 confers resistance to Magnaporthe oryzae. J Genet Genomics 38:209-216

Chen X, Shang J, Chen D, Lei C, Zou Y, Zhai W, Liu G, Xu J, Ling Z, Cao G, Ma B, Wang Y, Zhao X, Li S, Zhu L (2006) A B-lectin receptor kinase gene conferring rice blast resistance. Plant J 46:794-804

Conaway-Bormans CA, Marchetti MA, Johnson CW, McClung AM, Park WD (2003) Molecular markers linked to the blast resistance gene Pi-z in rice for use in marker-assisted selection. Theor Appl Genet 107:1014-1020 
Dai L, Wu J, Li X, Wang X, Liu X, Jantasuriyarat C, Kudrna D, Yu Y, Wing RA, Han B, Zhou B, Wang GL (2010) Genomic structure and evolution of the Pi2/9 locus in wild rice species. Theor Appl Genet 121:295-309

Deng Y, Zhu X, Shen Y, He Z (2006) Genetic characterization and fine mapping of the blast resistance locus Pigm(t) tightly linked to Pi2 and Pi9 in a broadspectrum resistant Chinese variety. Theor Appl Genet 113:705-713

Fjellstrom R, McClung AM, Shank AR (2006) SSR markers closely linked to the Pi-Z locus are useful for selection of blast resistance in a broad array of rice germplasm. Mol Breeding 17:149-157

Fukuoka S, Saka N, Koga H, Ono K, Shimizu T, Ebana K, Hayashi N, Takahashi A, Hirochika H, Okuno K, Yano M (2009) Loss of function of a proline-containing protein confers durable disease resistance in rice. Science 325:998-1001

Hayashi K, Hashimoto N, Daigen M, Ashikawa I (2004) Development of PCR-based SNP markers for rice blast resistance genes at the Piz locus. Theor Appl Genet 108:1212-1220

Hayashi K, Yoshida H (2009) Refunctionalization of the ancient rice blast disease resistance gene $P$ it by the recruitment of a retrotransposon as a promoter. Plant J 57:413-425

Hayashi K, Yoshida H, Ashikawa I (2006) Development of PCR-based allele-specific and InDel marker sets for nine rice blast resistance genes. Theor Appl Genet 113:251-260

Hayashi N, Inoue H, Kato T, Funao T, Shirota M, Shimizu T, Kanamori H, Yamane H, Hayano-Saito Y, Matsumoto T, Yano M, Takatsuji H (2010) Durable panicle blast-resistance gene Pb1 encodes an atypical CC-NBS-LRR protein and was generated by acquiring a promoter through local genome duplication. Plant J 64:498-510

Huang $H$, Huang L, Feng G, Wang S, Wang Y, Liu J, Jiang N, Yan W, Xu L, Sun P, Li Z, Pan S, Liu X, Xiao Y, Liu E, Dai L, Wang GL (2011) Molecular mapping of the new blast resistance genes Pi47 and Pi48 in the durably resistant local rice cultivar Xiangzi3150. Phytopathology 101:620-626

Jaiswal P, Ni J, Yap I, Ware D, Spooner W, Youens-Clark K, Ren L, Liang C, Zhao W, Ratnapu K, Faga B, Canaran P, Fogleman M, Hebbard C, Avraham S, Schmidt S, Casstevens TM, Buckler ES, Stein L, McCouch S (2006) Gramene: a bird's eye view of cereal genomes. Nucleic Acids Res 34(Database issue):D717-D723

Jeung JU, Kim BR, Cho YC, Han SS, Moon HP, Lee YT, Jena KK (2007) A novel gene, Pi40(t), linked to the DNA markers derived from NBS-LRR motifs confers broad spectrum of blast resistance in rice. Theor Appl Genet 115:1163-1177

Khush GS, Jena KK (2009) Current status and future prospects for research on blast resistance in rice (Oryza sativa L.). In: Wang GL, Valent B (eds) Advances in genetics, genomics and control of rice blast disease. Springer, Dordrecht, pp $1-10$

Kiyosawa S (1967) The inheritance of resistance of the zenith type varieties of rice to the blast fungus. Jap J Breed 17:99-107

Lee SK, Song MY, Seo YS, Kim HK, Ko S, Cao PJ, Suh JP, Yi G, Roh JH, Lee S, An G, Hahn TR, Wang GL, Ronald P, Jeon JS (2009) Rice Pi5-mediated resistance to Magnaporthe oryzae requires the presence of two coiled-coil-nucleotidebinding -leucine-rich repeat genes. Genetics 181:1627-1638

Lin F, Chen S, Que Z, Wang L, Liu X, Pan Q (2007) The blast resistance gene Pi37 encodes a nucleotide binding site-leucine-rich repeat proteinand is a member of a resistance gene cluster on rice chromosome 1. Genetics 177:1871-1880

Liu G, Lu G, Zeng L, Wang GL (2002) Two broad-spectrum blast resistance genes, Pig(t) and Pi2(t), are physically linked on rice chromosome 6. Mol Genet Genomics 267:472-480

Liu J, Wang X, Mitchell T, Hu Y, Liu X, Dai L, Wang GL (2010) Recent progress and understanding of the molecular mechanisms of the rice-Magnaporthe oryzae interaction. Mol Plant Pathol 11:419-427

Liu X, Lin F, Wang L, Pan Q (2007) The in silico map-based cloning of Pi36, a rice coiled-coil nucleotide-binding site leucine-rich repeat gene that confers racespecific resistance to the blast fungus. Genetics 176:2541-2549

Luo M, Wing AR (2003) An improved method for plant BAC library construction. Methods Mol Bio 236:3-20

McClung AM, Marchetti MA, Webb BD, Bollich CN (1997) Registration of 'Jefferson' rice. Crop Sci 37:629-630

Okuyama Y, Kanzaki H, Abe A, Yoshida K, Tamiru M, Saitoh H, Fujibe T, Matsumura H, Shenton M, Galam DC, Undan J, Ito A, Sone T, Terauchi R (2011) A multi-faceted genomics approach allows the isolation of rice Piablast resistance gene consisting of two adjacent NBS-LRR protein genes. Plant J 66:467-479

Ouyang S, Zhu W, Hamilton J, Lin H, Campbell M, Childs K, Thibaud-Nissen F, Malek RL, Lee Y, Zheng L, Orvis J, Haas B, Wortman J, Buell CR (2007) The
TIGR Rice Genome Annotation Resource: improvements and new features Nucleic Acids Res 35(Database issue):D883-D887

Qu S, Liu G, Zhou B, Bellizzi M, Zeng L, Dai L, Han B, Wang GL (2006) The broadspectrum blast resistance gene Pi9 encodes a nucleotide-binding siteleucine-rich repeat protein and is a member of a multigene family in rice. Genetics 172:1901-1914

Saghai-Maroof MA, Soliman KM, Jorgensen RA, Allard RW (1984) Ribosomal DNA spacer-length polymorphisms in barley: mendelian inheritance, chromosomal location, and population dynamics. Proc Natl Acad Sci 81:8014-8018

Sakata K, Nagamura Y, Numa H, Antonio BA, Nagasaki H, Idonuma A, Watanabe W, Shimizu Y, Horiuchi I, Matsumoto T, Sasaki T, Higo K (2002) RiceGAAS: an automated annotation system and database for rice genome sequence. Nucleic Acids Res 30:98-102

Shang J, Tao Y, Chen X, Zou Y, Lei C, Wang J, Li X, Zhao X, Zhang M, Lu Z, Xu J, Cheng Z, Wan J, Zhu L (2009) Identification of a new rice blast resistance gene, Pid3, by genome-wide comparison of paired nucleotide-binding site-leucine-rich repeat genes and their pseudogene alleles between the two sequenced rice genomes. Genetics 182:1303-1311

Sharma TR, Madhav MS, Singh BK, Shanker P, Jana TK, Dalal V, Pandit A, Singh A Gaikwad K, Upreti HC, Singh NK (2005) High-resolution mapping, cloning and molecular characterization of the Pi- $k^{h}$ gene of rice, which confers resistance to Magnaporthe grisea. Mol Genet Genomics 274:569-578

Shen Y, Adreit H, Zhu X, Milazzo J, Chen H, Tharreau D (2003) Resistance evaluation of some hybrid rice, conventional early indica and late japonica rice to Magnaporthe grisea in China. Scientia Agricultura Sinica 37:362-369

Skamnioti P, Gurr SJ (2009) Against the grain: safeguarding rice from rice blast disease. Trends Biotechnol 27:141-150

Takahashi A, Hayashi N, Miyao A, Hirochika H (2010) Unique features of the rice blast resistance Pish locus revealed by large scale retrotransposon-tagging. BMC Plant Biol 10:175

Talbot NJ (2003) On the trail of a cereal killer: exploring the biology of Magnaporthe grisea. Annu Rev Microbiol 57:177-202

Tang J, Zhu X, Wang Y, Liu L, Xu B, Li F, Fang J, Chu C (2011) Semi-dominant mutations in the CC-NB-LRR-type R gene, NLS1, lead to constitutive activation of defense responses in rice. Plant J 66:996-1007

Wang $Y$, Wang D, Deng X, Liu J, Sun P, Liu Y, Huang $H$, Jiang N, Kang H, Ning Y, Wang Z, Xiao Y, Liu X, Liu E, Dai L, Wang GL (2012) Molecular mapping of the blast resistance genes Pi2-1 and Pi51(t) in the durably resistant rice cultivar 'Tianjingyeshengdao'. Phytopathology 102:779-786

Wang ZX, Yano M, Yamanouchi U, Iwamoto M, Monna L, Hayasaka H, Katayose Y, Sasaki T (1999) The Pib gene for rice blast resistance belongs to the nucleotide-binding and leucine-rich repeat class of plant disease resistance genes. Plant J 19:55-64

Wu JL, Fan YY, Li DB, Zheng KL, Leung H, Zhuang JY (2005) Genetic control of rice blast resistance in the durably resistant cultivar gumei 2 against multiple isolates. Theor Appl Genet 111:50-56

Yang QZ, Lin F, Feng SJ, Wang L, Pan QH (2009) Recent progress on molecular mapping and cloning of blast resistance genes in rice (oryza sativa L.). Sci Agric Sin 42:1601-1615

Yuan B, Zhai C, Wang W, Zeng X, Xu X, Hu H, Lin F, Wang L, Pan Q (2011) The Pik-p resistance to Magnaporthe oryzae in rice is mediated by a pair of closely linked CC-NBS-LRR genes. Theor Appl Genet 122:1017-1028

Zhai C, Lin F, Dong Z, He X, Yuan B, Zeng X, Wang L, Pan Q (2011) The isolation and characterization of Pik, a rice blast resistance gene which emerged after rice domestication. New Phytol 189:321-334

Zhou B, Dolan M, Sakai H, Wang GL (2007) The genomic dynamics and evolutionary mechanism of the Pi2/9 locus in Rice. Mol Plant Microbe Interact 20:63-71

Zhou B, Qu S, Liu G, Dolan M, Sakai H, Lu G, Bellizzi M, Wang GL (2006) The eight amino-acid differences within three leucine-rich repeats between Pi2 and Piz- $t$ resistance proteins determine the resistance specificity to Magnaporthe grisea. Mol Plant Microbe Interact 19:1216-1228

Zhu X, Chen S, Yang J, Zhou S, Zeng L, Han J, Su J, Wang L, Pan Q (2012) The identification of Pi5O(t), a new member of the rice blast resistance Pi2/Pi9 multigene family. Theor Appl Genet 124:1295-1304

doi:10.1186/1939-8433-5-29

Cite this article as: Jiang et al:: Molecular mapping of the Pi2/9 allelic gene Pi2-2 conferring broad-spectrum resistance to Magnaporthe oryzae in the rice cultivar Jefferson. Rice 2012 5:29. 